were born prematurely. MRI was normal in 2, showed hypoplasia of the corpus callosum in 1, and a flattened sella turcica in 1. The EEG was normal, except for EMG contamination during attacks in 2. The EMG records had the same frequency as essential tremor. One had a positive family history of epilepsy. (Kanazawa O. Shuddering attacks-report of four children. Pediatr Neurol Nov 2000;23:421-424). (Respond: Dr Osamu Kanazawa MD PhD, Department of Pediatrics, Epilepsy Center, NishiNiigata Central National Hospital, I-14-I Masago, Niigata 950-2085, Japan).

COMMENT. Shuddering attacks (SA) are an uncommon benign disorder of infants and young children, with movements resembling shivering and straining, without impaired consciousness or epileptiform EEG, and showing resolution or improvement by 2 or 3 years of age. One previous report considered SAs an early manifestation of essential tremor (Vanasse $M$ et al. Neurology 1976;26:1027-30). They may be misdiagnosed as epilepsy.

Infantile tremor syndrome due to magnesium nutritional deficiency (Meningoencephalitic syndrome) should also be considered in differential diagnosis. This syndrome occurs in Indian infants between ages 6 months and 2 and $1 / 2$ years and is associated with severe malnutrition. The tremor is rapid and disappears in sleep. Serum, CSF, and urine magnesium levels are decreased. Tremors respond to magnesium, but a complicating delay in psychomotor development is not corrected. (Chaparwal BC et al. Dev Med Child Neurol 1980;22:252; Menkes JH, 1980).

\title{
PURPLE GLOVE SYNDROME WITH ORAL PHENYTOIN OVERDOSE
}

The occurrence of purple glove syndrome following the inadvertent oral administration of $1000 \mathrm{mg}$ phenytoin/day is reported in a 10-year-old $18 \mathrm{~kg}$ handicapped boy who was admitted in coma to the Niigata City General Hospital, Japan. Within a few hours of the initial overdose, the boy was drowsy and he had nystagmus and vomiting. Several hours later, he developed dark purple discoloration and marked swelling of his hands and feet. After 4 days of the continued treatment, the boy became comatose, and his mother discontinued the drug. On admission, and 2 days after discontinuing treatment, the blood phenytoin level was $78 \mathrm{mcg} / \mathrm{ml}$. The discoloration and swelling of the extremities gradually subsided and resolved completely after 11 days, without sequelae. (Yoshikawa H. Purple glove syndrome caused by oral administration of phenytoin. I Child Neurol Nov 2000;15:762). (Respond: Dr Hideto Yoshikawa, Department of Pediatrics, Niigata City General Hospital, 2-6-1 Shichikuyama, Niigata 950-8739, Japan).

COMMENT. Purple glove syndrome is a rare complication of intravenous administration of phenytoin. This appears to be the first report of the syndrome associated with oral phenytoin, administered in an overdose $(55 \mathrm{mg} / \mathrm{kg} / \mathrm{d})$. Both hands and feet were affected in a glove and sock distribution. Recovery was complete without sequelae.

\section{FAILED SURGERY FOR EPILEPSY}

Persistent or recurrent seizures occurring at least monthly are reported in $51(18 \%)$ of a series of 282 consecutive temporal resections for medically intractable epilepsy performed at Kings College Hospital, London, UK. Mean age at original surgery was 26 years (range 4 to 59 years), and the mean follow-up interval to reassessment was 6 years (range 3-17 yrs). Detailed assessment of postoperative seizures showed that of 20 with mesial temporal sclerosis (MTS), 14 (70\%) had seizures arising in the hemisphere of the resection, and $35 \%$ from the contralateral hemisphere. Of 10 patients with dysembryoplastic neuroepithelial 